\title{
Structural, electronic, and magnetic characteristics of $\mathrm{Np}_{2} \mathrm{Co}_{17}$
}

\author{
I. Halevy, ${ }^{1,2,3}$ A. Hen, ${ }^{2,4}$ I. Orion, ${ }^{2}$ E. Colineau, ${ }^{4}$ R. Eloirdi, ${ }^{4}$ J.-C. Griveau, ${ }^{4}$ P. Gaczyński, ${ }^{4}$ F. Wilhelm,,${ }^{5}$ A. Rogalev, ${ }^{5}$ \\ J.-P. Sanchez, ${ }^{6}$ M. L. Winterrose, ${ }^{3}$ N. Magnani, ${ }^{7}$ A. B. Shick, ${ }^{4,8}$ and R. Caciuffo ${ }^{4}$ \\ ${ }^{1}$ Physics Department, Nuclear Research Center Negev, P.O. Box 9001, IL84190 Beer-Sheva, Israel \\ ${ }^{2}$ Nuclear Engineering Department, Ben Gurion University, IL84105 Beer-Sheva, Israel \\ ${ }^{3}$ California Institute of Technology, W. M. Keck Laboratory 138-78, Pasadena, California 91125, USA \\ ${ }^{4}$ European Commission, Joint Research Centre, Institute for Transuranium Elements, Postfach 2340, DE-76125 Karlsruhe, Germany \\ ${ }^{5}$ European Synchrotron Radiation Facility (ESRF), Boîte Postale 220, FR-38043 Grenoble, France \\ ${ }^{6}$ SPSMS, UMR-E CEA/UJF-Grenoble 1, INAC, FR-38054 Grenoble, France \\ ${ }^{7}$ Lawrence Berkeley National Laboratory, Chemical Sciences Division, 1 Cyclotron Road, Berkeley, California 94720, USA \\ ${ }^{8}$ Institute of Physics, Academy of Sciences of the Czech Republic, Na Slovance 2, CZ-182 21 Prague, Czech Republic
}

(Received 30 November 2011; revised manuscript received 11 January 2012; published 30 January 2012)

\begin{abstract}
A previously unknown neptunium-transition-metal binary compound $\mathrm{Np}_{2} \mathrm{Co}_{17}$ has been synthesized and characterized by means of powder x-ray diffraction, ${ }^{237} \mathrm{~Np}$ Mössbauer spectroscopy, superconducting-quantuminterference-device magnetometry, and $\mathrm{x}$-ray magnetic circular dichroism (XMCD). The compound crystallizes in a $\mathrm{Th}_{2} \mathrm{Ni}_{17}$-type hexagonal structure with room-temperature lattice parameters $a=8.3107(1) \AA$ and $c=$ 8.1058(1) Å. Magnetization curves indicate the occurrence of ferromagnetic order below $T_{C}>350 \mathrm{~K}$. Mössbauer spectra suggest a $\mathrm{Np}^{3+}$ oxidation state and give an ordered moment of $\mu_{\mathrm{Np}}=1.57(4) \mu_{B}$ and $\mu_{\mathrm{Np}}=1.63(4) \mu_{B}$ for the Np atoms located, respectively, at the $2 b$ and $2 d$ crystallographic positions of the $P 6_{3} / \mathrm{mmc}$ space group. Combining these values with a sum-rule analysis of the XMCD spectra measured at the neptunium $M_{4,5}$ absorption edges, one obtains the spin and orbital contributions to the site-averaged $\mathrm{Np}$ moment $\left[\mu_{S}=-1.88(9) \mu_{B}\right.$, $\left.\mu_{L}=3.48(9) \mu_{B}\right]$. The ratio between the expectation value of the magnetic-dipole moment and the spin magnetic moment $\left(m_{\mathrm{md}} / \mu_{S}=+1.36\right)$ is positive as predicted for localized $5 f$ electrons and lies between the values calculated in intermediate-coupling (IC) and $j j$ approximations. The expectation value of the angular part of the spin-orbit-interaction operator is in excellent agreement with the IC estimate. The ordered moment averaged over the four inequivalent Co sites, as obtained from the saturation value of the magnetization, is $\mu_{\mathrm{Co}} \simeq 1.6 \mu_{B}$. The experimental results are discussed against the predictions of first-principles electronic-structure calculations based on the spin-polarized local-spin-density approximation plus the Hubbard interaction.
\end{abstract}

DOI: 10.1103/PhysRevB.85.014434 PACS number(s): 75.25.-j, 76.80.+y, 71.20.Lp, 71.15.Mb

\section{INTRODUCTION}

$R_{2} T_{17}$ ( $R=$ rare-earth metal, $T=$ transition metal) intermetallic compounds, such as $\mathrm{Sm}_{2} \mathrm{Co}_{17}$, have been extensively studied during the past decades, ${ }^{1,2}$ being widely used as high-performance permanent magnets in a number of devices. These compounds combine high magnetic anisotropy, arising from the localized rare-earth $4 f$ electrons, and high ordering temperature $T_{C}$, which is a consequence of the strong exchange interaction between transition-metal $3 d$ electrons. The intersublattice $R-T$ exchange interaction, which is known to increase when going from heavier to lighter $R \mathrm{~s}$, is another key parameter determining the magnetic behavior of these systems. Strong exchange interactions can enhance the contributions of excited states to the ground-state wave function ( $J$ mixing) with important consequences to the magnetic anisotropy. ${ }^{3-5}$ In compounds with light rare-earth ions, the $T$ and $R$ sublattices are coupled ferromagnetically, leading to high values of the spontaneous magnetization. For heavy $R \mathrm{~s}$ with their $4 f$ shells more than half filled, the two sublattices usually have antiparallel magnetic polarization, but discontinuous transitions to forced ferromagnetic or canted states can be induced by applying an external magnetic field. .,7 $^{6}$

$R_{2} T_{17}$ compounds crystallize either in the $P 6_{3} / m m c\left(D_{6 h}\right)$ space group with the $\mathrm{Th}_{2} \mathrm{Ni}_{17}$-type hexagonal structure (typical for heavy $R$ s including Y) or in the $R \overline{3} m$ space group with the $\mathrm{Th}_{2} \mathrm{Zn}_{17}$-type rhombohedral structure (typical for light $R \mathrm{~s}$ ).
In the former case, the unit cell contains two formula units with the $R$ atoms in the $2 b$ and $2 d$ positions and the $T$ atoms in the $4 f, 6 g, 12 k$, and $12 j$ positions. The structure can accommodate a relatively large number of substitutional or interstitial impurities, and it is known that the inclusion of nitrogen or carbon in the interstitial $6 h$ sites is accompanied by a substantial increase of the Curie temperature. ${ }^{8-11}$

The two $R$ sites in the $\mathrm{Th}_{2} \mathrm{Ni}_{17}$-type structure have a similar $T$-nearest-neighbor environment but a different nearest$R$ coordination. Nevertheless, they are often considered as magnetically equivalent ${ }^{6,12,13}$ as crystallographic disorder, which is not uncommon for the $R_{2} T_{17}$ phases, ${ }^{14}$ contributes to averaging the differences in the close surroundings of the two $R$ sites and provides a justification of this assumption.

Only a few actinide intermetallics with the hexagonal $\mathrm{Th}_{2} \mathrm{Ni}_{17}$ structure have been synthesized, namely, the ferromagnetic ternary $\mathrm{U}_{2} T_{17-y} X_{y}(T=\mathrm{Fe}, \mathrm{Co} ; X=\mathrm{Si}, \mathrm{Ge})$ compounds, ${ }^{15-18}$ a structural variant of the rhombohedral heavy-fermion antiferromagnet $\mathrm{U}_{2} \mathrm{Zn}_{17}{ }^{19-22}$ and the transuranium intermetallics, $\mathrm{Np}_{2} \mathrm{Ni}_{17},{ }^{23} \mathrm{Pu}_{2} \mathrm{Zn}_{17},{ }^{24}$ and $\mathrm{Pu}_{2} T_{17}(T=$ $\mathrm{Co}, \mathrm{Ni}) .{ }^{25,26}$ Extending these investigations to more actinide analogs is interesting as the wider radial extension of the $5 f$ electronic shell compared to the $4 f$ one should result in a larger intersublattice exchange interaction and in non-negligible An-An exchange coupling. The spin-orbit interaction in the $5 f$ configuration for the actinide atoms is also larger than the $4 f$ spin-orbit interaction. For these reasons, magnetic behavior 
even richer than that observed in the rare-earth compounds is expected. $^{27}$

Here, we report the results of a study performed to investigate the magnetic properties of the previously unknown $\mathrm{Np}_{2} \mathrm{Co}_{17}$ compound. By combining magnetization, Mössbauer, and x-ray magnetic-circular-dichroism (XMCD) measurements, we are able to determine the values of the magnetic moments of the two sublattices and to estimate orbital and spin contributions to the $\mathrm{Np}$ magnetic moment. The isotropic part of the spin-orbit interaction per hole of the $\mathrm{Np} 5 f$ states is determined from the branching ratio of the $\mathrm{x}-$ ray-absorption spectra at the $\mathrm{Np} M_{4,5}$ edges. The experimental value is very close to the result obtained theoretically in the intermediate-coupling (IC) approximation. The ratio between the magnetic-dipole term $\left\langle T_{z}\right\rangle$ and the spin component $\left\langle S_{z}\right\rangle$ deduced from the dichroic-signal-branching ratio also agrees well with the IC value.

The remainder of this paper is structured as follows. In Sec. II, we provide details on synthesis and experimental procedures. In Sec. III, we describe the crystal structure determined from a Rietveld refinement of the x-ray-diffraction pattern. The results of magnetization, Mössbauer, and XMCD measurements are reported and discussed in Sec. IV whereas the results of first-principles electronic-structure calculations are presented in Sec. V. A short summary and conclusions are found in Sec. VI.

\section{EXPERIMENTAL DETAILS}

Polycrystalline samples of $\mathrm{Np}_{2} \mathrm{Co}_{17}$ were prepared by arc melting stoichiometric amounts of high-purity elemental constituents $(99.9 \% \mathrm{~Np}, 99.999 \% \mathrm{Co})$ on a water-cooled copper hearth under an $\operatorname{Ar}(99.9999 \%)$ atmosphere. A Zr alloy was used as an oxygen getter. The weight losses during melting were less than $0.2 \%$. The samples were melted five times to improve the homogeneity, then wrapped in tantalum foils, and annealed at $800{ }^{\circ} \mathrm{C}$ in evacuated quartz ampoules for two weeks. Phase analysis of the obtained ingots was performed before and after annealing. Crystallographic analyses were performed at room temperature by $\mathrm{x}$-ray diffraction on samples with a mass of about $25 \mathrm{mg}$, finely ground and dispersed on a $\mathrm{Si}$ wafer. Data were collected in back-reflection mode with a Bruker D8 diffractometer and $\mathrm{Cu} K \alpha$ radiation selected by a $\mathrm{Ge}(111)$ monochromator. A one-dimensional positionsensitive detector was used to cover the angular range from 15 to $120^{\circ}$ with incremental steps of $0.0085^{\circ}$.

Magnetization and magnetic-susceptibility measurements were carried out in the temperature range between 2 and $350 \mathrm{~K}$ and in magnetic fields up to $7 \mathrm{~T}$ using a Quantum Design MPMS-7 superconducting-quantum-interference-device (SQUID) magnetometer. The ${ }^{237} \mathrm{~Np}$ Mössbauer spectra were recorded in a transmission-geometry spectrometer using the sinusoidal drive motion of a $\sim 100 \mathrm{mCi}{ }^{241} \mathrm{Am}$ metal source kept at a temperature of $4.2 \mathrm{~K}$. The absorber was prepared by grinding the sample to a fine powder to ensure a constant surface density with an optimal thickness of $140 \mathrm{mg}$ of $\mathrm{Np}$ per square centimeter. A liquid-helium cryostat was used to vary the sample temperature between $4.2 \mathrm{~K}$ and $77 \mathrm{~K}$. The velocity scale of the spectrometer was calibrated with reference to a $\mathrm{NpAl}_{2}$ reference sample $\left(B_{\mathrm{hf}}=330 \mathrm{~T}\right.$ at $\left.4.2 \mathrm{~K}\right)$.
The x-ray-absorption-spectroscopy (XAS) and XMCD experiments were carried out at the ID12 beamline of the European Synchrotron Radiation Facility (ESRF), which is dedicated to polarization-dependent spectroscopy in the photon-energy range from 2 to $15 \mathrm{keV}$. For the experiments at the $M_{4,5}$ edges of $\mathrm{Np}(3.6-3.9 \mathrm{keV})$, the source was the helical undulator Helios-II, which provided high flux of circularly-polarized x-ray photons with a polarization rate in excess of 0.95 . After monochromatization with a double-crystal-Si(111) monochromator, the rate of circular polarization reduced to about 0.42 at the $M_{5}$ edge and 0.5 at the $M_{4}$ absorption edge. For these measurements, we used a 9.51-mg sample glued with epoxy resin inside an Al holder with a $100-\mu \mathrm{m}$-thick Be window and $10-\mu \mathrm{m}$-thick kapton protection. The x-ray-absorption spectra were recorded using the total-fluorescence-yield detection mode in backscattering geometry for parallel $\mu^{+}(E)$ and antiparallel $\mu^{-}(E)$ alignments of the photon helicity with respect to a 7-T external magnetic field applied along the beam direction.

The x-ray-absorption spectra for right and left circularly polarized x-ray beams were then corrected for self-absorption effects and for incomplete circular polarization rates of incident $\mathrm{x}$-ray photons. The self-absorption corrections were done taking into account the chemical composition, the density, the practically infinite thickness of the bulky sample, the various background contributions (fluorescence of subshells and matrix as well as coherent and incoherent scattering), the angle of incidence of the x-ray beam, and finally the solid angle of the detector. ${ }^{28-30}$ The self-absorption corrections can be used with confidence as a measure of the absorption coefficient at the $M_{4,5}$ edges of $\mathrm{Np}$ since the spin-orbit coupling at these edges is large. They have been proven to work very well in the case of $U$ multilayers. ${ }^{31}$ The $\mathrm{Np}$ edge-jump intensity ratio $M_{5} / M_{4}$ was then normalized to $1: 2 / 3$ according to the statistical edge-jump ratio (defined as the ratio between the occupation numbers for the two spin-orbit-split core levels $j=3 / 2$ and 5/2). This statistical $\mathrm{Np}$ edge-jump intensity ratio $M_{5} / M_{4}$ is very close to the value (1.57) tabulated in the XCOM tables by Berger et al.in Ref. 32. A deviation of $\pm 10 \%$ in the $M_{5} / M_{4}$ XAS edge-jump normalization (ratio of 1.5) would affect the branching ratio $B$ by $\pm 2.5 \%$ and similarly the occupation numbers of the $j=5 / 2$ and $j=7 / 2$ subshells $n_{5 / 2}^{5 f}$ and $n_{7 / 2}^{5 f}$. The XMCD spectra $\mu^{+}-\mu^{-}$were obtained as the difference of the corrected x-ray-absorption spectra. To make sure that the final XMCD spectra are free of any experimental artifacts, measurements were also performed for the opposite direction of the applied magnetic field.

\section{CRYSTAL STRUCTURE}

The room-temperature powder-diffraction pattern of the investigated compound is shown in Fig. 1. The Rietveld analysis was performed with the X'PERT HIGHSCORE PLUS software package of PANalytical. The background was fitted with a polynomial, and the shape of the Bragg peaks was described by a pseudo-Voigt function.

The observed Bragg peaks can be indexed in the hexagonal $P 6_{3} / m m c$ space group with lattice parameters $a=$ 8.3107(1) $\AA$ and $c=8.1058(1) \AA$. Additional peaks reveal the presence of $\mathrm{NpO}_{2}$ as an impurity phase ( $\sim 3 \%$ in weight). 


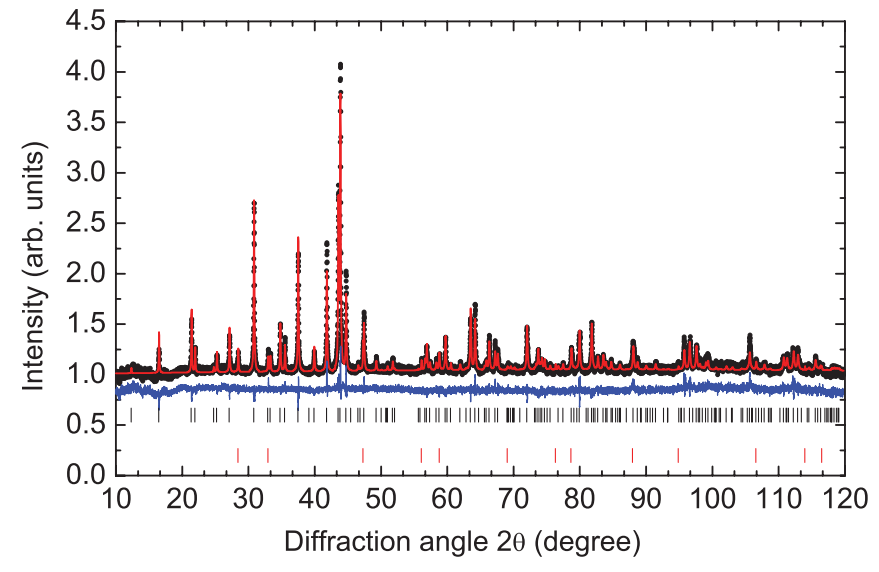

FIG. 1. (Color online) Observed (dots) and calculated (red line) $\mathrm{X}$-ray-diffraction patterns recorded at room temperature for $\mathrm{Np}_{2} \mathrm{Co}_{17}$. The lower trace (blue line) is the difference profile. The intensity distribution is plotted as a function of the full diffraction angle $2 \theta$ ( $\mathrm{Cu} K \alpha$ radiation). Vertical ticks indicate calculated angular positions of the Bragg peaks for the $\mathrm{Np}_{2} \mathrm{Co}_{17}$ phase (upper row, black) and the $\mathrm{NpO}_{2}$-impurity phase (lower row, red).

The best fit was obtained assuming full occupation of the sites corresponding to the ideal $\mathrm{Th}_{2} \mathrm{Ni}_{17}$-type structure, and any attempts to allow for the occupation of additional sites led to an increase of the weighted-pattern index $R_{\mathrm{wp}}$. The isotropic temperature factors $B_{\mathrm{DW}}$ have been fixed for all atoms. A final value $R_{\mathrm{wp}}=2 \%$ has been obtained. The structural parameters are reported in Table I. The crystal structure, shown in Fig. 2, consists of three different planes stacked perpendicularly to the $c$ axis in the sequence ABAC. Planes A contain Co atoms located at the $6 g$ and $12 k$ positions, and planes $\mathrm{B}$ and $\mathrm{C}$ contain both $12 j \mathrm{Co}$ atoms and $\mathrm{Np}$ at the $2 b$ and $2 d$ sites. The Co atoms at $4 f$ positions form a dumbbell arrangement separating the $\mathrm{A}, \mathrm{B}$, and $\mathrm{C}$ planes. The smallest nearest-neighbor $\mathrm{Np}-\mathrm{Np}$ distance is $>4 \AA$, well above the Hill limit of $\sim 3.2 \AA$ that separates neptunium compounds with localized $5 f$ states from itinerant systems. Appreciable crystal-field effects are therefore expected.

\section{PHYSICAL PROPERTIES}

\section{A. Experimental results}

The temperature $(T)$ and magnetic-field $\left(\mu_{0} H\right)$ dependence of the magnetization are shown in Fig. 3. The observed

TABLE I. Refined structural parameters for $\mathrm{Np}_{2} \mathrm{Co}_{17}$ at room temperature. The parameters refer to the hexagonal axes [space group $P 6_{3} / m m c, a=8.3107(1) \AA$, and $\left.c=8.1058(1) \AA\right]$. An isotropic Debye-Waller factor $B_{\mathrm{DW}}=0.5 \AA^{2}$ and full occupation were assumed for all sites.

\begin{tabular}{lccccc}
\hline \hline Atom & Site & Symmetry & $x$ & $y$ & $z$ \\
\hline $\mathrm{Np}_{1}$ & $2 b$ & $\overline{6} m 2$ & 0 & 0 & $1 / 4$ \\
$\mathrm{~Np}_{2}$ & $2 d$ & $\overline{6} m 2$ & $1 / 3$ & $2 / 3$ & $3 / 4$ \\
$\mathrm{Co}_{1}$ & $4 f$ & $3 m$ & $1 / 3$ & $2 / 3$ & $0.1048(5)$ \\
$\mathrm{Co}_{2}$ & $6 g$ & $2 / m$ & $1 / 2$ & 0 & 0 \\
$\mathrm{Co}_{3}$ & $12 j$ & $m$ & $0.3241(5)$ & $0.9547(3)$ & $1 / 4$ \\
$\mathrm{Co}_{4}$ & $12 k$ & $m$ & $0.1663(3)$ & $0.3326(6)$ & $0.9775(3)$ \\
\hline \hline
\end{tabular}

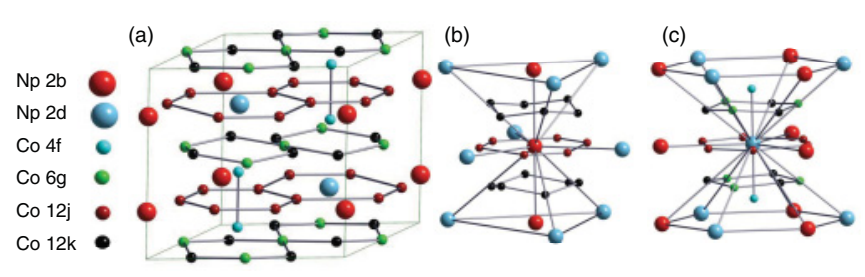

FIG. 2. (Color online) (a) Crystal structure of $\mathrm{Np}_{2} \mathrm{Co}_{17}$, corresponding to the $\mathrm{ABAC}$ stacking sequence characteristic of the ordered $\mathrm{Th}_{2} \mathrm{Ni}_{17}$-type structure. The coordination environment of the $\mathrm{Np}$ atoms located at sites $2 b$ and $2 d$ is shown in (b) and (c), respectively.

curves are typical for a soft ferromagnet with a vanishing coercive field. The magnetization at $20 \mathrm{~K}$ reaches a value $M_{S}=30.0(5) \mu_{B} /$ f.u. at $\mu_{0} H=7 \mathrm{~T}$. Saturation is achieved around $\mu_{0} H=1 \mathrm{~T}$ for all the explored temperatures up to $350 \mathrm{~K}$, the maximum value allowed by the device we used. This indicates a Curie temperature $T_{C}$ larger than $350 \mathrm{~K}$. Irreversibility associated with domain-wall motions is revealed by the splitting of the field-cooled (FC) and zero-field-cooled (ZFC) curves below $\sim 270 \mathrm{~K}$.

The $60 \mathrm{keV}$ electric-dipole ( $E 1)$ Mössbauer resonance of the ${ }^{237} \mathrm{~Np}$ nucleus provides a unique microscopic tool for gaining information on the electronic and magnetic properties of Np systems. ${ }^{33}$ Data recorded for $\mathrm{Np}_{2} \mathrm{Co}_{17}$ at $T=4.2 \mathrm{~K}$ are shown in Fig. 4 and compared with the calculated profile. The spectrum was analyzed using Lorentzian line shapes. The positions and relative intensities of the absorption lines of $\mathrm{Np}$ nuclei are calculated by solving the complete Hamiltonian for the hyperfine interactions in both the ground and excited nuclear state of ${ }^{237} \mathrm{~Np}$.

The spectrum is fully resolved and slightly asymmetric, indicating that magnetic and quadrupolar interactions are simultaneously present and that the former interaction dominates. As shown in Fig. 4, the spectrum is well fitted by considering two sets of hyperfine parameters corresponding to the two nonequivalent $\mathrm{Np}$ sites. Within experimental

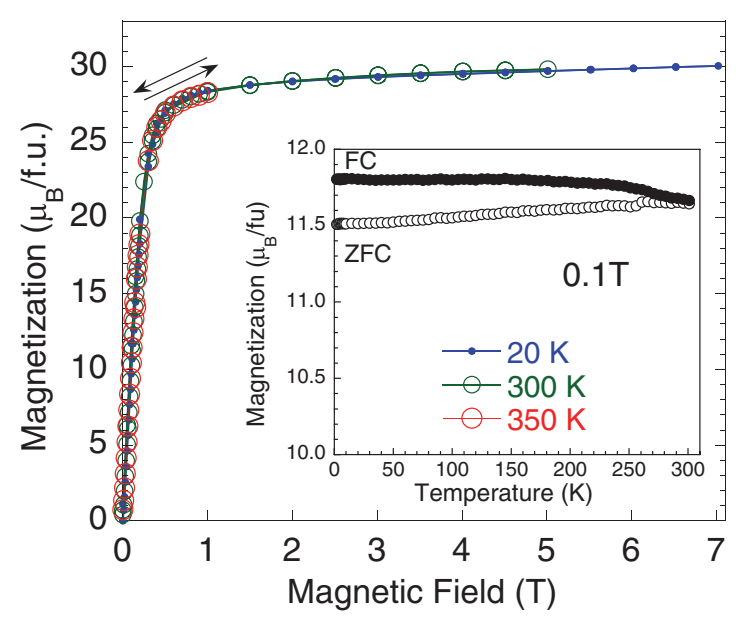

FIG. 3. (Color online) Magnetic-field dependence of the $\mathrm{Np}_{2} \mathrm{Co}_{17}$ magnetization measured on a polycrystalline sample at different temperatures from 20 to $350 \mathrm{~K}$. The inset shows the temperature dependence of the magnetization measured up to $300 \mathrm{~K}$ in a field $\mu_{0} H=0.1 \mathrm{~T}$ under field-cooled (FC) and zero-field-cooled (ZFC) conditions. 

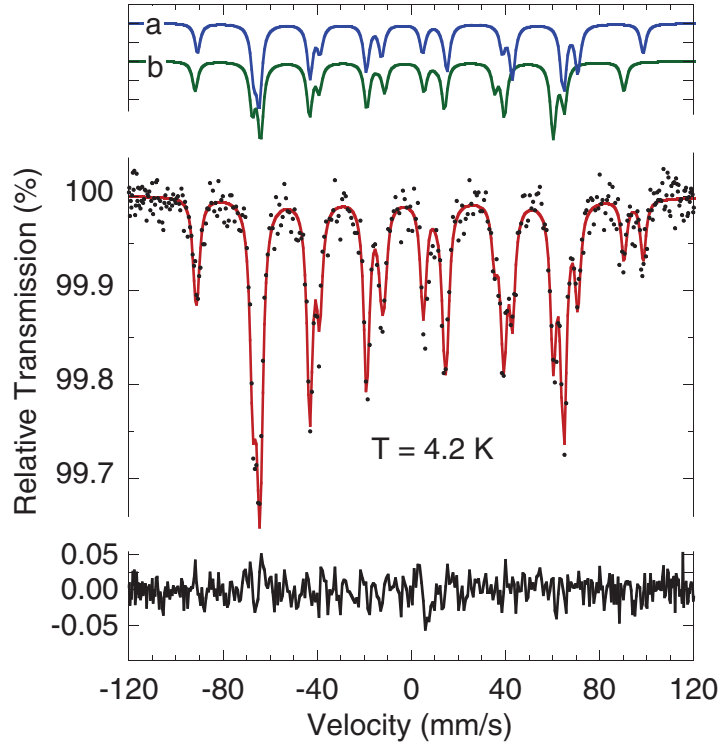

FIG. 4. (Color online) ${ }^{237} \mathrm{~Np}$ Mössbauer spectrum of $\mathrm{Np}_{2} \mathrm{Co}_{17}$ taken at $4.2 \mathrm{~K}$ in the ferromagnetic state. The solid red line represents the best fit to the data (dots) as given by the superposition of component spectra associated with the two inequivalent $\mathrm{Np}$ positions. The individual spectra, vertically shifted with respect to the base line, are shown as blue (a) and green (b) lines at the top of the figure. The lower trace (black line) is the difference profile. The fit slightly improves by adding the contribution from the $\mathrm{NpO}_{2}$ impurity, giving a single broad absorption line at $7.7 \mathrm{~mm} / \mathrm{s}$ where the difference profile shows a small dip.

uncertainty, the same relative intensity is observed at the two sites. Table II summarizes the best-fit parameters. The narrow linewidth $(W)$ confirms the absence of chemical disorder at the Np sites. On both sites, the main component ( $\left.\mathrm{eq} \equiv V_{z z}\right)$ of the electric-field gradient is taken collinear with the hyperfine field $\left(B_{\mathrm{hf}}\right)$, i.e., to the magnetization. Indeed, the $d-f$ exchange interaction is dominant in this compound and determines the quantization axis. ${ }^{34}$ The asymmetry parameter $\eta$ is set to zero in agreement with the axial symmetry of the Np sites. Slightly different isomer shifts $\left(\delta_{\text {IS }}\right)$ are observed, and the quadrupole coupling constants $\left(e^{2} q Q\right)$ are found to be negative. No temperature variation of the hyperfine parameters has been observed up to $77 \mathrm{~K}$. The $\mathrm{Np}$ ordered magnetic moment is calculated from the value of the hyperfine field through the relation $^{34} B_{\mathrm{hf}} / \mu_{\mathrm{Np}}=(215 \pm 5) \mathrm{T} / \mu_{B}$. The obtained average value, $\mu_{\mathrm{Np}}=1.60(4) \mu_{B}$, suggests a rather localized nature of the Np $5 f$ electrons in this compound.

XMCD is not only an element but also a shell-specific probe involving the excitation of core-level electrons to unoccupied states of a symmetry defined by the dipole selection rules of the optical transition. Sum-rule analyses ${ }^{35-38}$ allow one to separately determine orbital and spin contributions of these states to the total magnetic moment carried by the absorbing atom. XMCD spectra measured at the $M_{4,5}\left(3 d_{3 / 2,5 / 2} \rightarrow 5 f\right)$ $\mathrm{Np}$ absorption edges, with the sample kept at $50 \mathrm{~K}$ in a magnetic field of $7 \mathrm{~T}$, are shown in Fig. 5. The field dependence of the dichroic signal at the $M_{4}$ edge follows the curve obtained from SQUID measurements. The expectation value of the angular part of the valence-states spin-orbit operator $\langle\psi|\vec{\ell} \cdot \vec{s}| \psi\rangle$ is obtained from the XAS branching ratio, $B=$ $I_{M_{5}} /\left(I_{M_{5}}+I_{M_{4}}\right)$, as $^{39}$

$$
\frac{2\langle\vec{\ell} \cdot \vec{s}\rangle}{3 n_{h}}-\Delta=-\frac{5}{2}\left(B-\frac{3}{5}\right),
$$

where $I_{M_{4,5}}$ is the integrated intensity of the isotropic white line at the Np $M_{4,5}$ edges, $n_{h}$ is the number of holes in the $5 f$ shell, and $\Delta$ is a quantity dependent on the electronic configuration.

The orbital contribution to the magnetic moment is obtained from the dichroic signal integrated over the pair of spin-orbitsplit excitations, $\Delta I_{M_{5}}+\Delta I_{M_{4}}$, normalized to the isotropic $\mathrm{X}$-ray-absorption spectrum,

$$
\left\langle L_{z}\right\rangle=\frac{n_{h}}{I_{M_{5}}+I_{M_{4}}}\left(\Delta I_{M_{5}}+\Delta I_{M_{4}}\right) .
$$

A second sum rule correlates a linear combination of the partial dichroic signal $\Delta I_{M_{5}}$ and $\Delta I_{M_{4}}$ with the spin polarization $\left\langle S_{z}\right\rangle$,

$$
\left\langle S_{z}\right\rangle+3\left\langle T_{z}\right\rangle=\frac{n_{h}}{2\left(I_{M_{5}}+I_{M_{4}}\right)}\left(\Delta I_{M_{5}}-\frac{3}{2} \Delta I_{M_{4}}\right),
$$

where $T_{z}$ is the component along the quantization axis of the magnetic-dipole operator $\vec{T}=\sum_{i}\left[\vec{s}_{i}-3\left(\vec{r}_{i} \cdot \vec{s}_{i}\right) / r_{i}^{2}\right]$. This operator, correlating the spin and position of individual electrons, is associated with the asphericity of the electronic cloud, distorted by crystal-field or spin-orbit effects, and with the spin anisotropy. $\left\langle T_{z}\right\rangle$ is therefore correlated to the charge and magnetic anisotropy. ${ }^{40}$

The orbital and spin components of the total magnetic moment of the $5 f$ shell $\mu=-\left(\left\langle L_{z}\right\rangle+2\left\langle S_{z}\right\rangle\right)$ can then be obtained from XMCD spectra together with an estimate of $\left\langle T_{z}\right\rangle$ if the value of the total moment $\mu$ and the occupation number of the $5 f$ shell are known.

\section{B. Discussion of experimental results}

The Mössbauer spectrum in Fig. 4 clearly shows the presence of two inequivalent $\mathrm{Np}$ sites with magnetic moments $\mu_{\mathrm{Np}}=1.57(4) \mu_{B}$ and $\mu_{\mathrm{Np}}=1.63(4) \mu_{B}$. SQUID data provide a saturated magnetization of $30 \mu_{B}$ per formula unit, corresponding to an average magnetic moment of $\sim 1.58 \mu_{B}$ per Co atom if a ferromagnetic arrangement of the two sublattices is assumed. For a ferrimagnetic orientation, the

TABLE II. Estimated parameters for the ${ }^{237} \mathrm{~Np}$ Mössbauer spectra of $\mathrm{Np}_{2} \mathrm{Co}_{17}$ at $4.2 \mathrm{~K}$. $\delta_{\mathrm{IS}}$ is the isomer shift with respect to $\mathrm{NpAl}_{2}, B_{\mathrm{hf}}$

\begin{tabular}{|c|c|c|c|c|c|c|}
\hline Site & Intensity (\%) & $\delta_{\mathrm{IS}}(\mathrm{mm} / \mathrm{s})$ & $B_{\mathrm{hf}}(\mathrm{T})$ & $\mu_{\mathrm{Np}}\left(\mu_{B}\right)$ & $e^{2} q Q(\mathrm{~mm} / \mathrm{s})$ & $W(\mathrm{~mm} / \mathrm{s})$ \\
\hline$a(2 d)$ & 50 & $-13.7(2)$ & 351(1) & $1.63(4)$ & $-12.2(3)$ & $2.9(1)$ \\
\hline$b(2 b)$ & 50 & $-15.4(2)$ & $338(1)$ & $1.57(4)$ & $-4.5(3)$ & $2.9(1)$ \\
\hline
\end{tabular}
is the hyperfine field, $\mu_{\mathrm{Np}}$ is the ordered Np magnetic moment, $e^{2} q Q$ is the quadrupolar coupling constant, and $W$ is the full width at half maximum of individual spectral lines. 


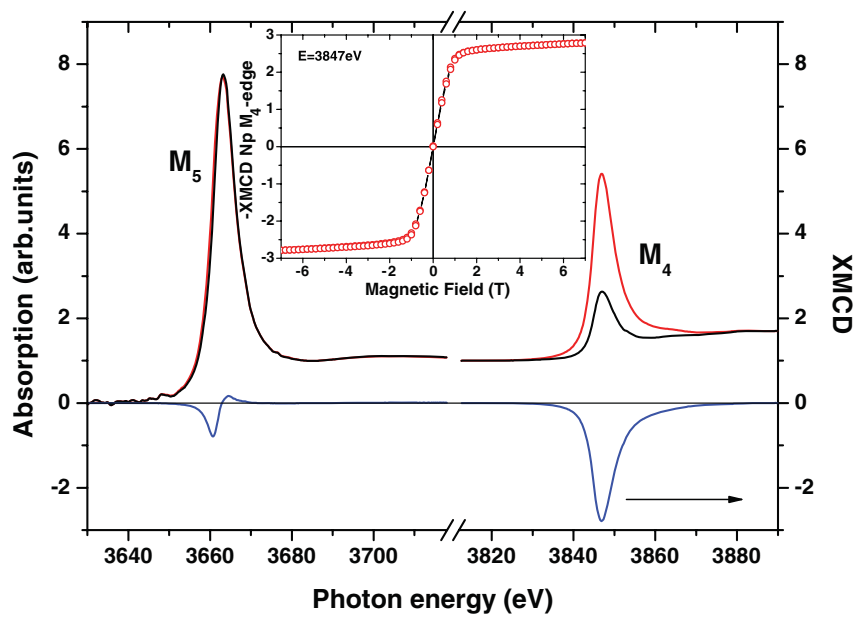

FIG. 5. (Color online) X-ray-absorption spectra $\mu^{+,-}$measured at $50 \mathrm{~K}$ at the $M_{4,5} \mathrm{~Np}$ absorption edges in $\mathrm{Np}_{2} \mathrm{Co}_{17}$; data have been measured with the photon helicity parallel (red line) and antiparallel (black line) to a 7-T magnetic field applied along the beam direction. The XMCD signal (blue line, lower trace) is given by $\mu^{+}-\mu^{-}$. The inset shows the field dependence of the XMCD signal at the $M_{4}$ edge.

average moment on the Co sites would be of $\sim 1.95 \mu_{B}$. The former estimate is much closer to the values reported for isostructural $R_{2} \mathrm{Co}_{17}(R=\mathrm{Y}, \mathrm{Lu}, \mathrm{Er})$ compounds in which the Co moment is in the range $1.62-1.65 \mu_{B},{ }^{41-44}$ suggesting that the $\mathrm{Np}$ in $\mathrm{Np}_{2} \mathrm{Co}_{17}$ behaves in a way similar to a light rare-earth metal. This is not surprising if one considers that according to the mechanism proposed in Ref. 45 , the $3 d$-spin moments of Co atoms are coupled antiferromagnetically to the $6 d$-spin moments of $\mathrm{Np}$ atoms. Due to the positive intra-atomic Hund's exchange coupling, the Np $5 f$-spin moments are in turn coupled parallel to the $\mathrm{Np} 6 d$-spin moment and are therefore antiparallel to the Co $3 d$ spins. On the other hand, the total $f$-electron moment (dominated by the angular component) is antiparallel to the spin-only contribution for a less-than-half-filled shell and parallel otherwise, leading to a ferromagnetic arrangement for the "light" lanthanides (and actinides) and a ferrimagnetic arrangement for the "heavy" lanthanides.

The isomer shifts $\delta_{\mathrm{IS}}=-13.7 \mathrm{~mm} / \mathrm{s}$ and $\delta_{\mathrm{IS}}=$ $-15.4 \mathrm{~mm} / \mathrm{s}$ obtained for the two Np sites are slightly smaller than the lower boundary of the range expected for a $\mathrm{Np}^{3+}$ charge state in a metallic environment $\left(-9<\delta_{\mathrm{IS}}<38 \mathrm{~mm} / \mathrm{s}\right)$ and close to the value corresponding to $\mathrm{Np}^{4+}$ free ions $\left(\delta_{\mathrm{IS}}=-9 \mathrm{~mm} / \mathrm{s}\right) .{ }^{46}$ For neptunium, the larger the electronic density at the nucleus, the smaller the $\delta_{\mathrm{IS}}$. As the presence of conduction electrons and partially delocalized $5 f$ electrons increases the electronic density at the Np nucleus, a substantial decrease of $\delta_{\text {IS }}$ is expected compared to the free-ion values. We therefore assume that the $\mathrm{Np}$ atoms in $\mathrm{Np}_{2} \mathrm{Co}_{17}$ are in a trivalent state with a $5 f^{4}$ electronic configuration. The occupied special positions, $2 b$ and $2 d$, have 18 and 20 Co nearest neighbors, respectively. In general the electronic density at the nucleus appears to diminish with increasing coordination number; thus, $\delta_{\text {IS }}$ will be larger (i.e., less negative) at the $2 d$ site. ${ }^{47}$ In addition to this, we expect the $3 d-5 f$ exchange interaction of the $\mathrm{Np}$ atoms to be larger for the site with more Co nearest neighbors. We therefore associate the moment of 1.57(4) $\mu_{B}$ to the $2 b$ site and the larger moment of 1.63(4) $\mu_{B}$ to the $2 d$ special position.

The magnetic moments and the quadrupolar coupling constants are strongly reduced with respect to the $\mathrm{Np}^{3+}$ freeion values $\left(\mu=2.57 \mu_{B}, e^{2} q Q \simeq-27.3 \mathrm{~mm} / \mathrm{s}\right)$. Assuming that the localized $5 f$-electron picture is correct, this reduction can be due to the combined effect of the $3 d-5 f$ exchange interaction and the crystal-field potential. The former is generally the strongest of the two ${ }^{41}$ and splits the ${ }^{5} I_{4}$ multiplet into nine singlets, the lowest in energy being the $J_{z}=4$ state, with the quantization direction for the $\mathrm{Np}$ sites parallel to the total moment of the Co sublattice. High-order terms in the crystal-field potential are expected to play an important role in determining the magnetic anisotropy as observed for other rare-earth intermetallic systems. ${ }^{48,49}$

As a positive sign of $V_{z z}^{\text {latt }}$ has been found for isostructural $R_{2} T_{17}$ systems $(R=\mathrm{Gd}$, Dy, Tm; $T=\mathrm{Co}, \mathrm{Ni}){ }^{50,51}$ an easy $c$-axis magnetization seems favored. This direction also coincides with the hexagonal symmetry axis; therefore, the only term which can change the ground-state composition is $B_{6}^{6} C_{6}^{(6)}$, which mixes the abovementioned $|+4\rangle$ state with the $|-2\rangle$. The coefficients of the wave function could then be determined by imposing the value of the ordered moment. This would give $0.5017|-2\rangle+0.8650+4\rangle$ as the ground state. The corresponding $5 f$-electron contribution to the quadrupolar interaction constant, proportional to $\left\langle 3 J_{z}^{2}-J(J+1)\right\rangle$, would be $e^{2} q^{5 f} Q=-18.5 \mathrm{~mm} / \mathrm{s}$. The total $e^{2} q Q$ is obtained by adding the lattice contribution $e^{2} q^{\text {latt }} Q\left(3 \cos ^{2} \theta-1\right) / 2$, proportional to the product of the quadrupole moment of the nucleus and the principal component of the lattice-electricfield gradient tensor $V_{z z}^{\text {latt }}$. The angle $\theta$ between the direction of the magnetic moment and the $c$ axis is not known as $T_{C}$ is too high, and the spectrum in the paramagnetic phase cannot be measured. Assuming $\theta=0$, from the experimental values given in Table II, one obtains $\sim 6 \mathrm{~mm} / \mathrm{s}$ and $\sim 14 \mathrm{~mm} / \mathrm{s}$ for $e^{2} q^{\text {latt }} Q$ at the $2 d$ and $2 b$ sites, respectively. This would indeed correspond to positive values for $V_{z z}^{\text {latt }}$ as found for isostructural $R_{2} T_{17}$ systems $(R=\mathrm{Gd}$, Dy, Tm; $T=\mathrm{Co}$, Ni) ${ }^{50,51}$ However, it must be stressed that the wave-function composition we used would require a very large value of $B_{6}^{6}$ so that the strong-exchange picture typical for lanthanide-cobalt intermetallic compounds would be in question and a more complex ground state could arise. Another possibility is that the magnetic-moment reduction be explained at least in part by an orbital-reduction factor, which would be a typical case for actinide atoms with partially delocalized electrons.

From Eq. (1), using the isotropic branching ratio $B=$ $0.757(5)$ provided by the XAS experiment, one obtains a $5 f$ spin-orbit interaction per hole of -0.392 , very close to the value calculated in the intermediate-coupling approximation for the $5 f^{4}$ electronic configuration. ${ }^{52}$ This supports the conclusion drawn from the Mössbauer isomer shift. Assuming $n_{h}=10, \Delta=-0.005$ (Ref. 38), and $\langle\psi|\vec{\ell} \cdot \vec{s}| \psi\rangle=-5.95$. One can then obtain the occupation numbers of the $j=5 / 2$ and $j=7 / 2$ subshells $n_{5 / 2}^{5 f}=3.4$ and $n_{7 / 2}^{5 f}=0.6$.

From the XMCD results, assuming $n_{h}=10$, one obtains a $5 f$-orbital component (neglecting the spd contributions) of the magnetic moment $\mu_{L}=3.48 \mu_{B}$ from Eq. (2). With a total moment $\mu_{\mathrm{Np}}=1.60 \mu_{B}$, this gives a spin contribution 
$\mu_{S}=-1.88 \mu_{B}$. It is noteworthy that $-\mu_{L} / \mu_{S} \simeq 1.85$ is close to the $\mathrm{Np}^{3+}$ free-ion value (1.90).

From Eqs. (2) and (3), one obtains the ratio $\left\langle L_{z}\right\rangle / 2\left(\left\langle S_{z}\right\rangle+\right.$ $\left.3\left\langle T_{z}\right\rangle\right)=-0.78$ between the orbital magnetic moment and an effective spin magnetic moment $2\left(\left\langle S_{z}\right\rangle+3\left\langle T_{z}\right\rangle\right)$. This corresponds to the ratio $3\left\langle T_{z}\right\rangle /\left\langle S_{z}\right\rangle=+1.36$, which differs by about a factor of 2 from the value $(+0.555)$ calculated for $\mathrm{Np}^{3+}$ in the intermediate-coupling scheme. ${ }^{37}$ The value of $\left\langle T_{z}\right\rangle$ is indeed expected to be non-negligible for localized $5 f$ systems in low symmetry. ${ }^{53}$

\section{ELECTRONIC-STRUCTURE CALCULATIONS}

To examine theoretically the electronic structure of $\mathrm{Np}_{2} \mathrm{Co}_{17}$ and to make a comparison with experimental data, we performed a spin-polarized local-spin-density approximation (LSDA) as well as LSDA plus Hubbard U (LSDA+U) calculations, using the experimental lattice parameters (Table I) for the ideal $\mathrm{Th}_{2} \mathrm{Ni}_{17}$-type structure. All calculations were performed making use of the in-house implementation ${ }^{54,55}$ of the full-potential linearized-augmented-plane-wave (FP-LAPW) method. This FP-LAPW version includes all relativistic effects: scalar-relativistic and spin-orbit coupling (SOC). The radii of the atomic "muffin-tin" (MT) spheres are set to 2.8 a.u. (Np) and 2.1 a.u. (Co). The basis-set size is characterized by the parameter $R_{\mathrm{Np}} K_{\max }=8.4$, and the Brillouin zone is sampled with $125 k$ points.

First, we apply the conventional spin-polarized LSDA, assuming antiparallel coupling between Co $3 d$ - and Np $5 f$ spin moments. ${ }^{45}$ Table III reports the calculated spin $\left(\mu_{S}\right)$, orbital $\left(\mu_{L}\right)$, and total $\left(\mu=\mu_{S}+\mu_{L}\right)$ magnetic moments in the muffin-tin spheres around $\mathrm{Np}$ and Co atoms (in $\mu_{B}$ units) together with the total values (including contributions from the interstitial region) per formula unit of $\mu_{S}, \mu_{L}$, and $\mu$. It is seen that the LSDA yields a substantially smaller value of the magnetization per formula unit $\left(22.8 \mu_{B}\right)$ than experimentally observed $\left(30 \mu_{B}\right)$.

Next, we apply LSDA $+\mathrm{U}$ calculations, making use of relativistic (including SOC) "around-mean-field" (AMF)LSDA+U (see Ref. 56). The Coulomb interaction in the Np $5 f$ shell is parameterized by Slater integrals $F_{0}=3.00 \mathrm{eV}, F_{2}=$ $7.43 \mathrm{eV}, F_{4}=4.83 \mathrm{eV}$, and $F_{6}=3.53 \mathrm{eV}$ as given in Ref. 52 .

TABLE III. Spin $\left(\mu_{S}\right)$, orbital $\left(\mu_{L}\right)$, and total $\left(\mu=\mu_{S}+\mu_{L}\right)$ magnetic moments in the "muffin-tin" spheres around the Np and Co atoms (in $\mu_{B}$ units) together with total values (including interstitial contributions) per formula unit of $\mu_{S}, \mu_{L}$, and $\mu$. Experimental values obtained from the Np Mössbauer spectra and SQUID-magnetization measurements are given in the last column.

\begin{tabular}{|c|c|c|c|c|c|c|c|c|}
\hline \multirow[b]{2}{*}{ Atom } & \multirow[b]{2}{*}{ Site } & \multicolumn{3}{|c|}{$\mathrm{LSDA}(+\mathrm{SOC})$} & \multicolumn{4}{|c|}{ AMF-LSDA+U } \\
\hline & & $\mu_{S}$ & $\mu_{L}$ & $\mu$ & $\mu_{S}$ & $\mu_{L}$ & $\mu$ & $\mu_{\text {expt. }}$ \\
\hline $\mathrm{Np}_{1}$ & $2 b$ & -3.15 & 1.97 & -1.18 & -1.96 & 3.50 & 1.54 & $1.57(4)$ \\
\hline $\mathrm{Np}_{2}$ & $2 d$ & -3.14 & 1.87 & -1.27 & -2.09 & 3.94 & 1.85 & $1.63(4)$ \\
\hline $\mathrm{Co}_{1}$ & $4 f$ & 1.58 & 0.05 & 1.63 & 1.59 & 0.05 & 1.64 & 1.58 \\
\hline $\mathrm{Co}_{2}$ & $6 g$ & 1.50 & 0.08 & 1.58 & 1.50 & 0.09 & 1.59 & 1.58 \\
\hline $\mathrm{Co}_{3}$ & $12 j$ & 1.38 & 0.10 & 1.48 & 1.37 & 0.13 & 1.50 & 1.58 \\
\hline $\mathrm{Co}_{4}$ & $12 k$ & 1.50 & 0.09 & 1.59 & 1.51 & 0.08 & 1.59 & 1.58 \\
\hline Total & & 17.56 & 5.26 & 22.82 & 20.14 & 9.12 & 29.26 & $30.0(5)$ \\
\hline
\end{tabular}

They correspond to commonly accepted values $U=3 \mathrm{eV}$ and $J=0.6 \mathrm{eV}$ for the Coulomb and exchange-interaction parameters, respectively.

Note that the LSDA+U yields the total magnetization in a very reasonable agreement with the experimental value, providing a good approximation for the values of the magnetic moment both at the $\mathrm{Np}$ and at the Co sites. The total electronenergy density of states (DOS) calculated for $U=3 \mathrm{eV}$ is shown in Fig. 6 together with the spin-resolved $d$-orbital projected DOS for Co atoms at the occupied lattice sites and $f$-orbital projected DOS for $\mathrm{Np}$ atoms at the $2 b$ and $2 d$ sites. The Co $d^{\uparrow}$ band is practically full whereas the Co $d^{\downarrow}$ band is
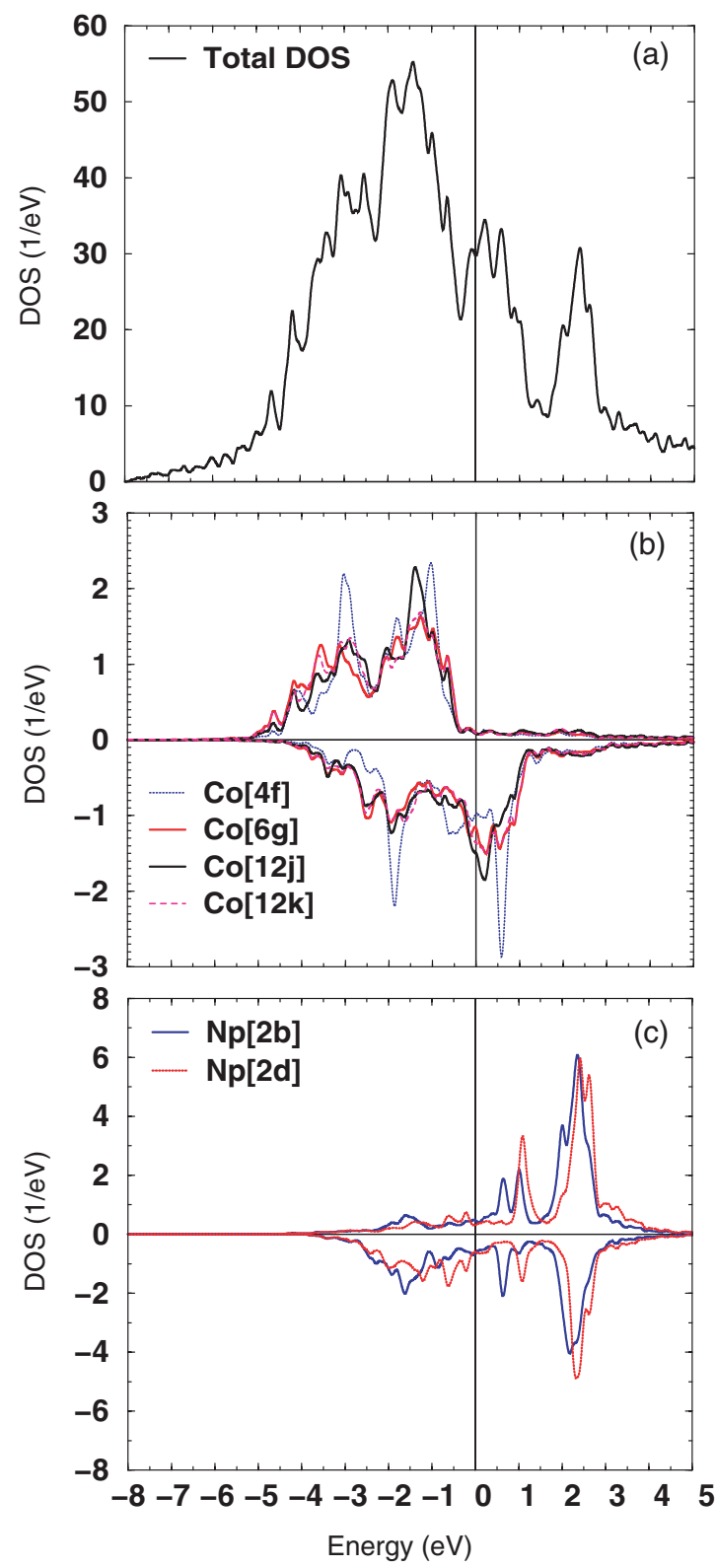

FIG. 6. (Color online) (a) The total electron-energy density of states (DOS) per formula unit for $\mathrm{Np}_{2} \mathrm{Co}_{17}$ calculated with relativistic $\mathrm{LSDA}+\mathrm{U}(U=3 \mathrm{eV})$. (b) The $d$-orbital projected DOS for Co atoms at $4 f, 6 g, 12 j$, and $12 k$ special positions of the $P 6_{3} / m m c$ space group. (c) The $f$-orbital projected DOS for Np atoms at $2 b$ and $2 d$ positions. 
TABLE IV. The $5 f$-state occupations $n^{5 f}, n_{5 / 2}^{5 f}$, and $n_{7 / 2}^{5 f}$; branching ratio $B ; 5 f$-electron contribution to the valence spin-orbit interaction per hole $w^{110} / n_{h}$; and the spin, orbital, and magnetic-dipole moments $\left(\mu_{S}^{5 f}, \mu_{L}^{5 f}\right.$, and $\left.m_{\mathrm{md}}\right)$ in Bohr magneton $\left(\mu_{B}\right)$ units.

\begin{tabular}{lccccccrrr}
\hline \hline Method & Atom[Site $]$ & $n^{5 f}$ & $n_{5 / 2}^{5 f}$ & $n_{7 / 2}^{5 f}$ & $B$ & $w^{110} / n_{h}$ & $\mu_{S}^{5 f}$ & $\mu_{L}^{5 f}$ & $m_{\text {md }}$ \\
\hline LSDA(+SOC) & $\mathrm{Np}[2 b]$ & 3.68 & 2.44 & 1.24 & 0.68 & -0.19 & -2.87 & 1.95 \\
LSDA(+SOC) & $\mathrm{Np}[2 d]$ & 3.72 & 2.44 & 1.28 & 0.68 & -0.19 & -2.85 & 1.85 \\
AMF-LSDA+U & $\mathrm{Np}[2 b]$ & 3.78 & 3.27 & 0.51 & 0.75 & -0.38 & -2.03 & 3.48 & -2.28 \\
AMF-LSDA+U & $\mathrm{Np}[2 d]$ & 3.82 & 3.31 & 0.51 & 0.75 & -0.38 & -2.12 & 3.76 & -2.44 \\
\hline \hline
\end{tabular}

partially occupied. The DOS peak in the vicinity of the Fermi level $\left(E_{F}\right)$ has mostly Co $d^{\downarrow}$ character. The occupied part of the Np $f$ manifold is mostly located in the energy range from $1 \mathrm{eV}$ to $3 \mathrm{eV}$ below $E_{F}$.

The $5 f$-electron contribution to the $\operatorname{spin}\left(\mu_{S}^{5 f}=-2\left\langle S_{z}^{5 f}\right\rangle\right)$ and orbital $\left(\mu_{L}^{5 f}=-\left\langle L_{z}^{5 f}\right\rangle\right)$ magnetic moments as well as the magnetic-dipole $m_{\mathrm{md}}=-6\left\langle T_{z}\right\rangle$ contribution are given in Table IV together with the calculated occupation numbers $\left(n^{5 f}, n_{5 / 2}^{5 f}\right.$, and $n_{7 / 2}^{5 f}$ ), the Np $M_{4,5}$-edge branching ratio $B$, and the valence spin-orbit interaction per hole $w^{110} / n_{h}=$ $2\langle\vec{\ell} \cdot \vec{s}\rangle_{5 f} / 3 n_{h}$ [from Eq. (1)]. These quantities can be directly compared with those deduced from the shell-specific XMCD spectra measured at the $\mathrm{Np} M_{4,5}$ absorption edges.

The $5 f$ occupation number, averaged over the two $\mathrm{Np}$ sites, is about 3.8, supporting the conclusions drawn from the analysis of the Mössbauer isomer shift. Reanalyzing the XMCD spectra for a number of $5 f$ holes $n_{h}=10.2$, one obtains $2\langle\vec{\ell} \cdot \vec{s}\rangle / 3 n_{h}=-0.39, \mu_{L}=3.55 \mu_{B}, \mu_{S}=-1.95 \mu_{B}$, and $m_{\mathrm{md}}=-2.59 \mu_{B}$. These values are in very reasonable agreement with the LSDA $+\mathrm{U}$ results. Moreover, the calculated ratios $\mu_{L} /\left(\mu_{S}+m_{\mathrm{md}}\right)=-0.81$ for $\mathrm{Np}[2 b]$ and -0.82 for $\mathrm{Np}[2 d]$ are in good quantitative agreement with the experimental value of -0.78 .

\section{CONCLUSIONS}

Combining magnetization-, Mössbauer-, and XMCDspectroscopy measurements, we have investigated the magnetic properties of $\mathrm{Np}_{2} \mathrm{Co}_{17}$, one of the few transuranium intermetallic compounds crystallizing in the hexagonal
$\mathrm{Th}_{2} \mathrm{Ni}_{17}$-type structure. The compound orders ferromagnetically above room temperature with a saturation magnetization $M_{\text {sat }}=30 \mu_{B} /$ f.u. and a small, easy-axis magnetic anisotropy. The total magnetic moment at the Np sites has been determined from the hyperfine field provided by Mössbauer spectroscopy whereas the spin and orbital components of the magnetic moment have been obtained from XMCD measurements at the $\mathrm{Np} M_{4,5}$ absorption edges. Although $\mathrm{Np}_{2} \mathrm{Co}_{17}$ adopts the hexagonal crystal structure typical of heavy rare earths, the observed magnetic order is typical for light rare-earth isostructural analogs. The comparison between experimental data and first-principles electronic-structure calculations demonstrates the necessity to go beyond the local-spin-density approximation to correctly describe the magnetic properties of this family of $\mathrm{Np}$ compounds and that a satisfactory level of accuracy is obtained already by fully relativistic $\mathrm{LSDA}+\mathrm{U}$ approaches.

\section{ACKNOWLEDGMENTS}

We thank D. Bouëxière and G. Pagliosa for their technical support and G. H. Lander for help during the XMCD experiment at ESRF. The high-purity neptunium metal required for the fabrication of the sample was made available through a loan agreement between Lawrence Livermore National Laboratory (LLNL) and ITU in the frame of a collaboration involving LLNL, Los Alamos National Laboratory, and the US Department of Energy. A.H. acknowledges the European Commission for support in the frame of the Training and Mobility of Researchers program. The support from Czech Republic Grants No. GACR P204/10/0330, No. GAAV IAA100100912, and No. AV0Z10100520 is thankfully acknowledged.
${ }^{1}$ K. Kumar, J. Appl. Phys. 63, R13 (1988).

${ }^{2}$ J. M. D. Coey, Rare-Earth Iron Permanent Magnets (Clarendon Press, Oxford, 1996).

${ }^{3}$ O. Moze, R. Caciuffo, H.-S. Li, B.-P. Hu, J. M. D. Coey, R. Osborn, and A. D. Taylor, Phys. Rev. B 42, 1940 (1990).

${ }^{4}$ L. Pareti, A. Paoluzi, and N. Magnani, J. Magn. Magn. Mater. 251, 178 (2002).

${ }^{5}$ N. Magnani, S. Carretta, G. Amoretti, L. Pareti, A. Paoluzi, R. Caciuffo, and J. A. Stride, Appl. Phys. Lett. 85, 4097 (2004).

${ }^{6}$ M. D. Kuz'min, Y. Skourski, K. P. Skokov, and K.-H. Müller, Phys. Rev. B 75, 184439 (2007).
${ }^{7}$ A. V. Andreev, Y. Skourski, M. D. Kuz'min, S. Yasin, S. Zherlitsyn, R. Daou, J. Wosnitza, A. Iwasa, A. Kondo, A. Matsuo, and K. Kindo, Phys. Rev. B 83, 184422 (2011).

${ }^{8}$ J. Coey and H. Sun, J. Magn. Magn. Mater. 87, L251 (1990).

${ }^{9}$ H. Sun, J. M. D. Coey, V. Otani, and D. P. R. Hurley, J. Phys.: Condens. Matter 2, 6465 (1990).

${ }^{10}$ J. P. Woods, B. M. Patterson, A. S. Fernando, S. S. Jaswal, D. Welipitiya, and D. J. Sellmyer, Phys. Rev. B 51, 1064 (1995).

${ }^{11}$ R. F. Sabirianov and S. S. Jaswal, J. Appl. Phys. 79, 5942 (1996).

${ }^{12}$ K. Clausen and B. Lebech, J. Phys. C 15, 5095 (1982).

${ }^{13}$ P. C. M. Gubbens and K. H. J. Buschow, J. Phys. F 12, 2715 (1982). 
${ }^{14}$ O. Moze, R. Caciuffo, B. Gillon, G. Calestani, F. E. Kayzel, and J. J. M. Franse, Phys. Rev. B 50, 9293 (1994).

${ }^{15}$ T. Berlureau, B. Chevalier, L. Fournes, and J. Etourneau, Mater. Lett. 9, 21 (1989).

${ }^{16}$ T. Berlureau, P. Gravereau, B. Chevalier, and J. Etourneau, J. Solid State Chem. 104, 328 (1993).

${ }^{17}$ B. Chevalier, P. Gravereau, T. Berlureau, L. Fournes, and J. Etourneau, J. Alloys Compd. 233, 174 (1996).

${ }^{18}$ Y. Shiokawa, M. Hara, Y. Haga, R. Amano, and M. Takahashi, J. Alloys Compd. 213, 513 (1994).

${ }^{19}$ E. S. Makarov and S. I. Vinogradov, Kristallografiya 1, 650 (1956) [Sov. Phys. Crystallogr. 1, 499 (1956)].

${ }^{20}$ A. Iandelli and A. Palenzona, J. Less-Common Met. 12, 333 (1967).

${ }^{21}$ Q. Johnson, G. S. Smith, and D. H. Wood, Acta Crystallogr. B 25, 464 (1969).

${ }^{22}$ H. R. Ott, H. Rudigier, P. Delsing, and Z. Fisk, Phys. Rev. Lett. 52, 1551 (1984).

${ }^{23}$ M. Akabori, R. G. Haire, J. K. Gibson, Y. Okamoto, and T. Ogawa, J. Nucl. Mater. 247, 240 (1997).

${ }^{24}$ E. M. Cramer and D. H. Wood, J. Less-Common Met. 13, 112 (1967).

${ }^{25}$ O. J. C. Runnals, Can. J. Chem. 34, 133 (1956).

${ }^{26}$ D. Poole, M. Bale, P. Mardon, J. Marples, and J. Nichols, in Plutonium 1960, edited by E. Grison, W. Lord, and R. Fowler (Cleaver-Hume Press Ltd., London, 1961), p. 267.

${ }^{27}$ P. Santini, S. Carretta, G. Amoretti, R. Caciuffo, N. Magnani, and G. H. Lander, Rev. Mod. Phys. 81, 807 (2009).

${ }^{28}$ J. Goulon, C. Goulon-Ginet, R. Cortes, and J. M. Dubois, J. Phys. (Paris) 43, 539 (1982).

${ }^{29}$ L. Tröger, D. Arvanitis, K. Baberschke, H. Michaelis, U. Grimm, and E. Zschech, Phys. Rev. B 46, 3283 (1992).

${ }^{30}$ P. Pfalzer, J.-P. Urbach, M. Klemm, S. Horn, M. L. denBoer, A. I. Frenkel, and J. P. Kirkland, Phys. Rev. B 60, 9335 (1999).

${ }^{31}$ F. Wilhelm, N. Jaouen, A. Rogalev, W. G. Stirling, R. Springell, S. W. Zochowski, A. M. Beesley, S. D. Brown, M. F. Thomas, G. H. Lander, S. Langridge, R. C. C. Ward, and M. R. Wells, Phys. Rev. B 76, 024425 (2007).

${ }^{32}$ M. J. Berger, J. H. Hubbell, S. M. Seltzer, J. Chang, J. S. Coursey, R. Sukumar, D. S. Zucker, and K. Olsen (2010), [http://physics.nist.gov/xcom].

${ }^{33}$ J. P. Sanchez, E. Colineau, P. Vulliet, and K. Tomala, J. Alloys Compd. 275, 154 (1998).
${ }^{34}$ B. D. Dunlap and G. M. Kalvius, in Handbook on the Physics and Chemistry of the Actinides, edited by A. J. Freeman and G. H. Lander (North-Holland, Amsterdam, 1985), Vol. 2, p. 329.

${ }^{35}$ B. T. Thole, P. Carra, F. Sette, and G. van der Laan, Phys. Rev. Lett. 68, 1943 (1992).

${ }^{36}$ P. Carra, B. T. Thole, M. Altarelli, and X. Wang, Phys. Rev. Lett. 70, 694 (1993).

${ }^{37}$ G. van der Laan and B. T. Thole, Phys. Rev. B 53, 14458 (1996).

${ }^{38}$ G. van der Laan, K. T. Moore, J. G. Tobin, B. W. Chung, M. A. Wall, and A. J. Schwartz, Phys. Rev. Lett. 93, 097401 (2004).

${ }^{39}$ B. T. Thole and G. van der Laan, Phys. Rev. A 38, 1943 (1988).

${ }^{40}$ S. P. Collins, D. Laundy, C. C. Tang, and G. van der Laan, J. Phys.: Condens. Matter 7, 9325 (1995).

${ }^{41}$ K. H. J. Buschow, Rep. Prog. Phys. 40, 1179 (1977).

${ }^{42}$ B. Matthaei, J. J. M. Franse, S. Sinnema, and R. J. Radwanski, J. Phys., Colloq. 49, 533 (1988).

${ }^{43}$ E. A. Tereshina and A. V. Andreev, J. Magn. Magn. Mater. 320, e132 (2008).

${ }^{44}$ O. Moze, R. Caciuffo, B. Gillon, and F. E. Kayzel, J. Magn. Magn. Mater. 104-107, 1394 (1992).

${ }^{45}$ M. Brooks, O. Eriksson, and B. Johansson, J. Phys.: Condens. Matter 1, 5861 (1989).

${ }^{46}$ G. M. Kalvius, J. Gal, L. Asch, and W. Potzel, Hyperfine Interact. 72, 77 (1992).

${ }^{47}$ F. J. van Steenwijk, H. T. Lefever, R. C. Thiel, and K. H. J. Buschow, Physica B+C (Amsterdam) 92, 52 (1977).

${ }^{48}$ O. Moze, R. M. Ibberson, R. Caciuffo, and K. H. J. Buschow, J. Less-Common Met. 166, 329 (1990).

${ }^{49}$ R. Caciuffo, G. Amoretti, K. H. J. Buschow, O. Moze, A. P. Murani, and B. Paci, J. Phys.: Condens. Matter 7, 7981 (1995).

${ }^{50}$ P. C. M. Gubbens, A. M. van der Kraan, and K. H. J. Buschow, J. Magn. Magn. Mater. 54-57, 483 (1986).

${ }^{51}$ P. C. M. Gubbens, A. M. van der Kraan, J. J. van Loef, and K. H. J. Buschow, J. Magn. Magn. Mater. 67, 255 (1987).

${ }^{52}$ K. T. Moore and G. van der Laan, Rev. Mod. Phys. 81, 235 (2009).

${ }^{53}$ P. Carra, Synchrotron Radiat. News 5, 21 (1992).

${ }^{54}$ A. B. Shick, D. L. Novikov, and A. J. Freeman, Phys. Rev. B 56, 14259(R) (1997).

${ }^{55}$ A. B. Shick and W. E. Pickett, Phys. Rev. Lett. 86, 300 (2001).

${ }^{56}$ A. B. Shick, V. Drchal, and L. Havela, Europhys. Lett. 69, 588 (2005). 